\title{
Intra-abdominal hypertension and acute pancreatitis
}

\author{
Mifkovic A, Skultety J, Sykora P, Prochotsky A, Okolicany R \\ 2nd Department of Surgery, Faculty of Medicine, Comenius University, Bratislava, Slovakia. \\ mifkovic@gmail.com
}

\begin{abstract}
Intra-abdominal hypertension (IAH) contributes to organ dysfunction and leads to the development of the abdominal compartment syndrome (ACS). IAH and ACS are relatively frequent findings in patiens with severe acute pancreatitis (SAP) and are associated with deterioration in organ functions. The most affected are cardiovascular, respiratory and renal functions. The incidence of IAH in patients with SAP is approximately $60-80 \%$. There is an accumulating evidence in human and animal studies that changes of perfusion, particularly to the microvasculature, are crucial events in the progression of acute pancreatitis (AP). The perfusion of the small and large intestine is impaired due to reduced arterial pressure, increased vascular resistence and diminished portal blood flow. Bacterial translocation has been described in patients with ACS, and this may apply to patients with SAP. Approximately $30-40 \%$ of SAP patients develop ACS because of pancreatic (retroperitoneal) inflammation, peripancreatic tissue edema, formation of fluid collections or abdominal distension. Surgical debridement was the preferred treatment to control necrotizing pancreatitis in the past. However, the management of necrotizing pancreatitis has changed over the last decade. The main objective of this article is to describe the association between IAH and AP and to emphasize this situation in clinical praxis as well (Fig. 1, Ref. 38). Full Text in PDF www.elis.sk.

Key words: intra-abdominal hypertension, acute pancreatitis, organ dysfunction, abdominal compartment syndrome.
\end{abstract}

The first description of an elevated intra-abdominal pressure (IAP) was recorded more than 132 years ago, when Marey and Burt demonstrated the relationship between the intra-abdominal pressure and respiratory function (23).

The compartment syndrome is a condition in which an increased pressure in a confined anatomical space adversely affects circulation and threatens the perfusion of tissues. The abdominal cavity is a specific space in the human body. Any change in the volume of any of its contents will elevate the IAP (2). IAH contributes to organ dysfunction and leads to the development of ACS. The incidence of IAH in patients with SAP is approximately 60$80 \%$ (18). ACS is defined as a state of serious organ dysfunction resulting from sustained increase of IAP, that affects especially the cardiovascular, respiratory and renal system (1). ACS is characterized by multiple organ dysfunction syndrome (MODS) which can results in death (2). Organ dysunction typically includes hemodynamic instability, respiratory insufficiency with an impaired gas exchange, and acute renal failure with oliguria as the most prominent signs early in the course of the disease. SAP is a serious disease with a mortality of $25-40 \%$. Patients with SAP tend to have aelevated IAP, which can lead to IAH and subsequently to ACS. About $11-40 \%$ of SAP patients suffer from complications of ACS $(3,15)$. SAP patiets with ACS complications tend to have a mortality rate of $10-75 \%(3,15,18)$.

2nd Department of Surgery, Faculty of Medicine, Comenius University, Bratislava, Slovakia

Address for correspondence: A. Mifkovic, MD, $\mathrm{PhD}$, 2nd Department of Surgery LFUK, Antolská 11, SK-85107 Bratislava 5, Slovakia.

Phone: +421.903182928
IAP is highly variable in normal individuals and depends on body mass index and the position of the body $(24,25,26)$.

\section{Definitions}

Many conditions are reported in association with IAH and ACS, and they can be classified into four following categories. First group - conditions that decrease abdominal wall compliance. Second group - conditions that increase intraluminal contents, third group - conditions related to abdominal collections of fluid, air or blood. Fourth group - conditions related to capillary leak and fluid resuscitation (22).

For a long time, IAH and ACS were considered as a problem occuring only in surgical patients, but later they have been identified as a cause of organ dysfunction in several patients without an apparent abdominal surgical conditions (such as burn and sepsis patients). This category of ACS has been defined as secondary ACS. Primary $A C S$ refers to ACS due to an intra-abdominal cause, and ACS associated with SAP is a common cause of the primary ACS (18).

\section{IAH and $A C S$}

An increased IAP has been shown to affect the function of organ systems both within and outside of the abdominal cavity. The central concept in the understanding of IAH-induced organ dysfunction is the observation that the increased IAP is partly transmitted to the thorax as a consequence of cephalad displacement of the diaphragm. This leads to an increased intrathoracic pressure (ITP) and ultimately to an increased jugular venous pressure, decreased venous return from the brain, and possibly an increased intracranial pressure in patients at risk of intracranial hyperten- 
sion. IAH also has profound effects on the respiratory system, most importantly inducing a decreased functional residual capacity of the lungs and decreased compliance of the thoracic wall. These changes can result in the clinical signs of the secondary (or extrapulmonary) acute respiratory distress syndrome (ARDS), which requires advanced ventilatory strategies (22).

Malbrain et al. (2006) reported that IAH at admission was associated with organ dysfunction in a mixed medical-surgical ICU (intensive care unit) population, and that the occurrence of IAH during ICU admission was an independent predictor of mortality (4).

In the strictest sense, only IAP values ranging from sub-atmospheric to zero $\mathrm{mmHg}$ can be considered normal. However, certain physiological characteristics, such as morbid obesity, can be associated with a chronic increased IAP to which the patient has adapted, and the clinical significance of mildly or moderately elevated values needs to be assessed in view of the initial ,steady state" of the individual patient. For example, it has been demonstrated that an increased abdominal diameter in morbidly obese patients is associated with an elevated IAP in the absence of other significant pathophysiology $(26,27,28)$.

The following definitions were developed by a multi-disciplinary panel of critical care physicians and nurses during the second WSACS (World Society of Abdominal Compartment Syndrome) meeting. These definitions are based partly on published evidence and partly on the opinion of relevant experts (4):

"IAP is the steady-state pressure concealed within the abdominal cavity ". It increases with diaphragmatic contraction (during inspiration) and decreases with diaphragmatic relaxation (during expiration). IAP is affected by the volume of solid organs and the intestines, space-occupying lesions (ascites, blood, tumours), and the extensibility of the abdominal wall.

"IAH is defined by a sustained or repeated pathological elevation in IAP $>12 \mathrm{mmHg}$ ". This cut-off value was chosen because organ dysfunction becomes manifest in the majority of patients at an IAP of $12 \mathrm{mmHg}$ or higher.

"The abdominal perfusion pressure is calculated as the difference between the mean arterial pressure and IAP". It considers both arterial inflow and restrictions to venous outflow and may thus be the best predictor of damage caused by IAH.

"IAP should be expressed in $\mathrm{mmHg}$ and measured at endexpiration in the complete supine position after ensuring that abdominal muscle contractions are absent and with the transducer zeroed at the level of the midaxillary line. The reference standard of intermittent IAP measurement is via the bladder with a maximal instillation volume of $25 \mathrm{ml}$ sterile saline ". It has been found necessary to establish IAP monitoring because physical examination is not accurate enough to predict IAH. The bladder technique has achieved a widespread adoption due to its simplicity and noninvasiveness, whereas the direct and continuous peritoneal IAP measurement still requires further clinical validation.

"ACS is defined as a sustained IAP $>20 \mathrm{mmHg}$ (with or without abdominal perfusion pressure $<60 \mathrm{mmHg}$ ) that is associated with a new organ dysfunction or failure ". IAH is of higher significance than the absolute increase in IAP. Comorbidities (chronic renal, pulmonary and cardiac disease) may aggravate the deleterious effects of IAH and lower the threshold value of IAP at affected organs.

Currently, there is no evidence suggesting that the standard measurement techniques for IAP could not be applicable and reliable in patients with SAP. The most commonly used technique is the bladder pressure measurement through a Foley catheter (33).

\section{Acute pancreatitis}

AP has an annual incidence of 5-40 per 100000 population (5) with an overall mortality rate approaching $1-5$ per 100000 population (5). Approximately one-third of patients develop pancreatic necrosis, which has an associated mortality rate approaching $30 \%$ (6). This severe form of the disease is characterized by pancreatic necrosis, cytokine activation, systemic inflammatory response syndrome (SIRS) and MODS. Necrotic pancreatic and peripancreatic tissue may become infected, further increasing morbidity and mortality (7). Pancreatic perfusion and hypoxia have a significant impact on the early stages of disease and play an important role in the pathogenesis of pancreatic necrosis (8). There is anaccumulating evidence in human and animal studies that changes of perfusion, particularly to the microvasculature, are significant events in the progression of AP. Microvascular compromise has been suggested as the critical event in the development of SAP and treatment aimed at the microvasculature may decrease necrosis and disease severity (9). Interestingly, recently accumulated data indicates that the innervation of pancreas may also play an important role in the initiation and maintenance of pancreatic inflammation (10).

AP is described as having a wide range of severity from a mild, self-limiting condition to a fulminant illness resulting in death within a few days of onset. Mortality in AP has two peaks. The first peak is caused by SIRS, which takes place in the first week. Sepsis accounts for the second peak. It begins 1 to 3 weeks after the onset of pancreatitis and is caused by pancreatic superinfection. The origin of this infection arises from translocation of intestinal bacteria. The disruption of gut mucosal barrier during AP contributes to the genesis of this infection (11). Microcirculation disturbance is one of the main causes of injuries of the pancreas and other organs during AP. The gastrointestinal tract is one of the most frequently affected organs. Sepsis due to infected pancreatic necrosis is the most serious complication in late phase of SAP and contributes to the high mortality rate of this disease. This complication is thought to be the result of the bacterial translocation from the gastrointestinal tract. Bacterial translocation plays an important role for infectious complications in SAP. The alteration of intestinal mucosal integrity may increase intestinal permeability and may be implicated in bacterial translocation. It is suggested that an increase in intestinal permeability is correlated with the changes of tight junction and/or apoptosis in intestinal epithelial cells. The intestinal mucosa forms an effective barrier to the intraluminal bacteria and toxins, as well as absorbs the nutrient. The mechanisms underlying gut barrier dysfunction in AP are complex and still not fully elucidated. In early phase of AP, gut mucosa hypoperfusion resulting from hypovolemia and systemic inflammatory response causes intestinal muscosal damage and is the possible reason of a high intestinal permeability and 
absorptive capacity disruption. Early intestinal mucosal damage may play an important role in the pathophysiology of SAP, and it has long been involved in the development of sepsis and multiple organ failure as the major cause of death in $\operatorname{AP}(2,35)$. SAP is a difficult clinical condition, which prognosis has not significantly improved until now. One of the reasons my be that the treatment strategies do not fully aim at the key event of AP (12).

\section{Pathophysiology of ACS}

The knowledge on the pathophysiology of IAH and ACS in past has been obtained from experimental animal studies (rats, rabbits and pigs). The porcine model seems to come closest to ACS in humans. IAH impairs venous return from the periphery to the right heart. Simultaneously, it increases left ventricular afterload. Both effects lead to a diminished cardiac output, lower arterial blood pressure and lower organ perfusion pressure. The impairment of cardiac function causes arrhythmias, especially in pre-existing ischaemic heart disease. In critically ill patients, these events may increase the need for catecholamines with further negative consequences for hemodynamic stability. IAH displaces the diaphragm cranially and thus impairs thoracic compliance. This leads anatomically to basal atelectases of the lungs and, functionally, to a restrictive ventilation disorder. Oxygenation is further diminished by mismatch of ventilation and perfusion. Increased pressure is thus required for mechanical ventilation, which further lowers venous return and the mean arterial pressure. The elevated intrathoracic pressure increases jugular venous pressure, which may result in an increased intra-cranial pressure and cerebral oedema $(2,13)$. Most syndromes are preceded by a prodromal phase during which a number of nonspecific symptomps and signs appear. The ACS is no exception to this general rule, and IAH represents the prodromal phase of ACS (26). Oliguria is one of the first clinical signs of IAH. The mechanisms responsible for the impaired renal function include decreased venous outflow, reduced arterial perfusion and a direct compression of the renal parenchyma. Under these circumstances, the renin-angiotensin system is activated, which results in the retention of sodium and water. The perfusion of the small and large intestine is impaired due to the reduced arterial pressure, increased vascular resistence and diminished portal blood flow. This will result in the hypomotility of the gut and swelling of the intestinal walls. The translocation of luminal bacteria into sterile compartments of the portal system is facilitated during this condition. Under extreme conditions, ischaemic necrosis of the gut and liver can occur $(2,13)$. The gut appears to be particularly sensitive to IAH with virtually all intra-abdominal and retroeritoneal organs demonstrating a decreased blood flow in the presence of elevated IAP (29). The perfusion of lower extremities can also be compromised by an increased IAP. The moderately elevated IAH reduces femoral venous blood flow, whereas a markedly elevated IAP has been described as reducing or even stopping femoral artery flood flow. The reduced oxygen supply to the splanchnic area favours anaerobic metabolism and consequently leads to lactic acidosis. The profound detrimental effects of IAH on the macroand microcirculation of various body compartments augment each other and thus result in a vicious cycle (13). The IAP of $20 \mathrm{mmHg}$ diminishes intestinal mucosal perfusion and has been speculated as a possible mechanism for subsequent development of bacterial translocation, sepsis, and MODS (30).

Moreover, the infection of pancreatic necrosis, another much feared complication for patients with SAP, may be related to increased IAP. Animal studies have shown an increased rate of bacterial translocation in AP in those with increased IAP (19). Bacterial translocation has been described in patients with ACS, and this may apply to patients with SAP (18). Recent clinical studies provided new evidence for the association between elevated IAP and infection. In one study, pancreatic infection occurred in $60 \%$ of patients with ACS, whereas it was rare in patients with lower IAP(2). In another study, the maximum IAP was $19 \mathrm{mmHg}$ in patients who developed an infected pancreatic necrosis, whereas it was $11 \mathrm{mmHg}$ in patients with an uncomplicated course. Therefore, it is likely that IAH is involved to some extent in both development of necrosis and infection of the necrosis, the two major determinants of outcome (20).

Jochenberger et al (2009) in their prospective close-cohort study compared the course of arginine vasopressin (AVP) and copeptin plasma concentrations between patients with infection, severe sepsis, and septic shock. The authors concluded that severe sepsis induced a stronger AVP response than an infection without systemic inflammation. However, the lack of a difference in AVP plasma concentrations between patients with and without shock indicated that the AVP system did not function normally in severe sepsis. Their data supported the hypothesis that the impaired AVP response is at least partially responsible for the failure to restore vascular tone in septic shock (36). Copeptin, the C-terminal part of the AVP precursor peptide, was found to be the stable and sensitive surrogate marker for AVP release. Copeptin behaves in a similar manner to mature AVP in the circulation, with respect to osmotic stimuli and hypotension. During past years, copeptin measurement has been shown to be of interest in a variety of clinical indications, including cardiovascular diseases such as heart failure, myocardial infarction, and stroke (37). Copeptin levels were found to closely mirror the production of AVP. It has been shown that copeptin more subtly mirrors the individual stress level compared to cortisol. Due to the positive association of copeptin with the severity of illness and outcome, copeptin has been proposed as a prognostic marker in an acute illness. The prognostic accuracy of copeptin has been analysed in sepsis, pneumonia, lower respiratory tract infections, stroke and other acute illnesses. Copeptin improves the prognostic information provided by commonly used clinical scoring instruments (38). The role of copeptin in patients with AP and IAH is not clear yet. The realisation of new controlled prospected trials is necessary in order to explain the role of copeptin in these pathological situations.

The complex relationship between IAP and organ dysfunction, the prevalence of IAH and ACS in various patient populations, the true progression of IAH to ACS following a systemic injury, and the optimal management strategy for this multifaceted pathophysiologic process have yet to be fully elucidated and tested. We are clearly far from the end of the process in understanding IAH and ACS, but rather very much at the beginning. Although sepsisinduced organ failure is a frequent cause of IAH/ACS, and IAH/ 
/ACS may well contribute to the subsequent development of sepsis, this association is infrequently recognized by many clinicians (31).

\section{Abdominal compartment syndrome during acute pancreatitis}

Approximately $30-40 \%$ of SAP patients develop ACS because of pancreatic retroperitoneal inflammation, peripancreatic tissue oedema, formation of fluid collections or abdominal distension. This subsequently leads to intestinal ischaemia with ileus and renal failure. ACS generally affects cardiac, pulmonary and renal functions, and contributes to MODS with a mortality rate $10-50 \%$ within two weeks. The degree of IAP in patients with SAP seems to correlate with the degree of organ dysfunction, the severity of the disease, the length of the stay at the intensive care unit and mortality (15). The development of ACS in patients with SAP occurs most commonly in the early course of the disease and is likely caused by the combined effects of the aggressive fluid resuscitation and inflammatory process in the retroperitoneum leading to the development of visceral edema and pancreatic ascites (32).

The under-diagnosed and untreated ACS is a potential contributing factor to the development of early organ failure seen in patients with SAP and warrants a routine measurement of IAP in all patients treated for SAP. The current estimate of the prevalence of IAH in SAP is about $40 \%$, with about $10 \%$ overall progressing to ACS associated with increased hospital mortality rates. In the majority of cases, the development of IAH is rapid and mainly due to the combined effects of aggressive fluid resuscitation and the inflammatory process in the retroperitoneum leading to the development of visceral edema and pancreatic ascites within days or even hours from admission. Athough in some cases a delayed form of ACS has been associated with an emergence of infected peripancreatic necrosis (31).

At a later stage, complications related to pancreatitis and pancreatic necrosis, such as pancreatic abscess or pseudocysts, or bleeding from venous or arterial origin, may cause IAH. In these che ases, tmonitoring of IAH may help in the an early detection of new intra-abdominal pathology (18).

\section{Diagnostics and measurement of IAP}

The clinical sign of a "tense abdomen" is not sensitive enough to diagnose IAH and ACS. When bladder pressure was taken as the gold standard of IAP measurement, the sensitivity of physical examination in the diagnosis of IAH was reported to be as low as $40 \%$. In patients at risk, it is thus necessary to obtain objective data on IAP preferably by an uncomplicated and non-invasive technique. The pressure in the urinary bladder has been found to reflect IAP with the accuracy sufficient for clinical purpose. The 'bladder technique' has been widely adopted due to its simplicity and non-invasiveness. In the presence of IAH, the serial IAP measurements should be the prefered technique for measurement. In the WSACS consensus paper (4), the technique of measuring IAP continually by transduction of intra-vesical pressure has been defined. For the assessment of the time-course of IAP in an individual patient, it is very important to obtain measurements in exactly the same conditions (in analogy to central venous pressure). This highlights the importance of the zero reference point (the midaxillary line at the iliac crest) and the vesical instillation volume of saline $(25 \mathrm{ml})$, which avoids the measurement of falsely elevated IAP with higher volumes (13).

The early diagnosis of ACS and its adequate management is crucial. The measurement and monitoring of IAP via a urinary bladder catheter is a simple procedure, which requires virtually no technical skills and little resources. However, this procedure is invasive and is associated with a significant discomfort for the patient. It has also been shown that indwelling urinary catheters are associated with a higher incidence of infectious complications and prevalence of nosocomial pathogens. Clearly, the placement of a urinary catheter should not be routinely recommended for all patients, especially not for those who are unlikely to develop ACS. Therefore, clinical assessment in selecting the patients that are likely to develop ACS is of particular importance. The development of IAH and ACS during the AP could be predicted by the use of clinical multifactorial scoring systems (APACHE II, MODS, Glasgow-Imrie score), thus allowing a timely and appropriate selection of patients for this invasive procedure during the first hours and days of the disease. The clinical scores of patiens who eventually suffered from IAH were higher during the first days in comparison to the group of patiens with normal IAP (16).

Al-Bahrani et al (2008) in their study evaluated the predictive CT (computer tomography) features of intra-abdominal hypertension in critically ill patients. The authors reporting the CT examination of critically ill patients should consider the two features indicative of IAH. The first is the round belly sign and bowel wall thickening with enhancement. This CT feautures can be useful also in patients with SAP (Fig. 1).

\section{Management of IAH in patients with SAP}

IAH is clearly related to organ dysfunction in AP, and because it is reversible and to some extent preventable, IAH should definitely be considered a therapeutic target. IAP should be measured regularly in patients with SAP, at least every 4 hours or whenever the clinical condition of the patient deteriorates (21). The nonsur-

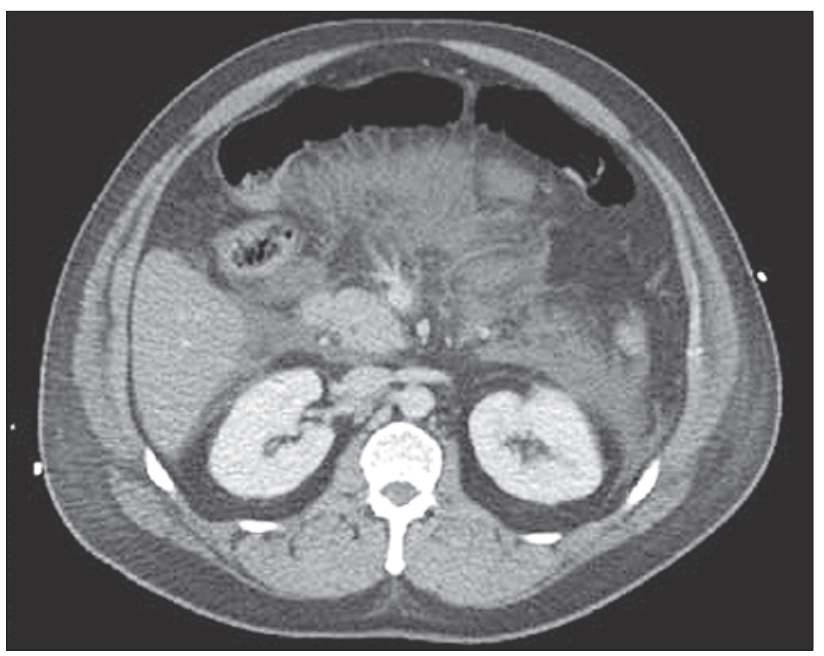

Fig. 1. Computed tomography scan showing round belly sign. 
gical measures to reduce IAP should be considered first, and when ineffective, the surgical approaches may be necessary in patients with a persistent organ dysfunction (18). The treatment of ACS in patients with SAP is bassed on the recognition of the principal cause of the IAH. If an ultrasound examination confirms the presence of large volumes of pancreatic ascites, the first line treatment would be the percutaneous drainage of the intraperitoneal exudates, which can lead to an significant drop in IAP (34).

Prevention with judicious use of crystalloids is important, and nonsurgical interventions, such as nasogastric decompression, short-term use of neuromuscular blockers, removal of fluid by extracorporeal techniques, and percutaneous drainage of ascites should be instituted early. The indications for surgical decompression are still not clearly defined, but undoubtedly, some patients benefit from it. It can be achieved with full-thickness laparotomy (midline or transverse subcostal) or through a subcutaneous linea alba fasciotomy. Despite the improvement in physiological variables and significant decrease in IAP, the effects of surgical decompression on organ function and outcome are less clear. Because of the significant morbidity associated with the surgical decompression and the management of the ensuing open abdomen, more research is needed to define better the appropriate indications and techniques for surgical intervention (18). Cardiovascular dysfunction and failure are commonly encountered in the patients with IAH or ACS. An accurate assessment and optimalization of preload, contractility and afterload is essential in restoring organ perfusion and function of these patients (31).

The surgical debridement was the preferred treatment in controlling necrotizing pancreatitis in past. However, the management of necrotizing pancreatitis has changed since the last decade. The first approach now tends to be non-surgical and relies on conservative strategies including an early transfer of patients to the intensive care units at specialized centres. The indication for necrosectomy is still given in cases of infected necrosis as well as intestinal infarction, perforation or bleeding, but there is a clear trend towards surgical treatment as late and as rarely as possible. In contrast, more and more studies are published promoting the decompression laparotomy for SAP patients developing ACS defined by IAP greater than $20 \mathrm{mmHg}$ associated with new organ failure. This procedure can not only prevent a critical decrease of intestinal and renal perfusion, but may lead to an improvement of the respiratory situation $(2,14)$.

The standard treatment after exhausting the conservative management options is the decompressive midline laparotomy. Although the percutaneous drainage of pancreatic ascites, when present, can sometimes be helpful, at least as a temporizing method. The midline decompressive laparotomy is effective in decreasing IAP, rapid and easy to perform, but it is associated with a high risk of intestinal fistulas, and in many cases, failure to close the fascia requires a complex reconstructive surgery 9-12 months later. The subcutaneous approach eliminates the open abdomen, but might not be effective enough. In addition, the subcutaneous fasciotomy always results in a ventral hernia requiring repair later on. Clinical experience also shows that an early closure of the open abdomen is accompanied with an improved clinical, nutritional and infection situation when the "catabolic drain" of the open wound is closed (1).
The percutaneous drainage of large amounts of pancreatic ascites may decrease the IAP considerably and represents the first line of treatment if appropriate. In most cases, however, the surgical decompresion through a vertical midline incision without exploring the pancreas further is the most effective and safest procedure. The decompression performed 2-3 weeks after the onset of the disease can be combined with necrosectomy. The primary fascial closure of the abdominal wall following the abdominal decompresion can be attempted, but in most cases the prolonged inflammatory process in the abdomen and the risk of recurrent ACS favors the use of gradual closure or delayed reconstruction of the abdominal wall (31).

Hong Chen et al. (2008) in their study concluded that IAH and ACS is a frequent complication in patients admitted to the ICU because of AP. Patiens with IAP at approximately 10-12 mmHg and early signs of changes in physiologic variables should be seriously considered for an urgent decompresion to improve the survival (2).

No therapeutic option should ever be excluded from a potential consideration in patiens with ACS. The current standard of care would now suggest the need for intervention at an IAP of 20-25 $\mathrm{mmHg}$ rather than $35-40 \mathrm{mmHg}$ that was commonly accepted 15 years ago (31).

\section{Conclusion and challenges for the future}

IAH and ACS are now recognized as being dynamic, rather than static, processes characterised by a constantly changing continuum of physiological events. IAH and ACS do not apper suddenly, but rather develop over the time in response to cellular ischaemia and reperfusion injury (31). IAH is a frequent problem in critically ill patients, and it causes significant morbidity with a potentially fatal outcome when left untreated (22). AP patients are at risk for IAH and ACS because of the large volume of intra-abdominal and peripancreatic inflammatory fluid collection, capillary leakage caused by an increased permeability, bowel and splanchnic edema, resuscitation fluid and other factors (2). IAH and ACS are relatively frequent findings in patiens with SAP and are associated with a deterioration in organ functions. Especially affected are the cardiac, renal and respiratory functions. The intraabdominal pressure correlates with the severity of organ failure. The high admission IAP is associated with a prolonged intensive care stay (17).

In looking to future, we must begin by recognizing that IAH and ACS may occur in virtually all patient populations, irrespecive of age, illness, or injury. The future of IAH and ACS must begin with educating clinicians of all disciplines as to the widespread presence, morbidity, and associated mortality of elevated IAP, IAH, and ACS within their patients 'populations. To facilitate the communication regarding IAH and ACS and to allow a meaningful comparison of clinical trials, it is imperative that a common terminology and data set are adopted for future discussions and research. Future research must be focused on performing rigorous, prospective, multi-center, human trials to answer the numerous questions that remain. Additionally, there is a need for more widespread publishing of articles on IAH and ACS throughout the scientific literature in order to more effectively educate clinicians who may encounter these disease processes (31). 


\section{References}

1. Leppäniemi A, Mentula P, Hienonen P, Kemppainen E. Transverse laparostomy is feasible and effective in the treatment of abdominal compartment syndrome in severe acute pancreatitis. World J Emerg Surg 2008; DOI:10.1186/1749-7922-3-6.

2. Chen H, Li F, Sun JB, Jia JG. Abdominal compartment sydrome in patiens with severe acute pancreatitis in early stage. World J Gastroenterol 2008; 14 (22): 3541-3548.

3. Zhang MJ, Zhang GL, Yuan WB, Ni J, Huang LF. Treatment of abdominal compartment syndrome in severe acute pancreatitis patients with traditional chinese medicine. World J Gastroenterol 2008; 14 (22): 3574-3578.

4. Malbrain ML, Cheatham ML, Kirkpatrick A et al. Results from the international conference of experts on intra-abdominal hypertension and abdominal compartment syndrome. I. Definitions. Intens Care Med 2006; 32: 1722-1732.

5. Toouli J, Brooke-Smith M, Bassi C, Carr-Locke D, Telford J, Freeny P et al. Guidelines for the management of acute pancreatitis. J Gastroenterol Hepatol 2002; 17 (Suppl): S15-S39.

6. Mitchell RM, Byrne MF, Baillie J. Pancreatitis. Lancet 2003; 361 : 1447-1455.

7. Perez A, Whang EE, Brooks DC, Moore FD Jr, Hughes MD, Sica GT et al. Is severity of necrotizing pancreatitis increased in extended necrosis and infected necrosis? Pancreas 2002; 25: 229-233.

8. Kinnala PJ, Kuttila KT, Gronroos JM, Havia TV, Nevalainen TJ, Niinikoski JH. Pancreatic tissue perfusion in experimental acute pancreatitis. Eur J Surg 2001; 167: 689-694.

9. Plusczyk T, Witzel B, Menger MD, Schilling M. ETA and ETB receptor function in pancreatitis-associated microcirculatory failure, inflammation, and parenchymal injury. Am J Physiol Gastrointest Liver Physiol 2003; 285: G145-G153.

10. Liddle RA. The role of Transient Receptor Potential Vanilloid 1 (TRPV1) channels in pancreatitis. Biochim Biophys Acta 2007; 1772 (8): 869-878.

11. Lutgendorff F, Nijmeijer RM, Sandström PA, Trulsson LM, Magnusson KE et al. Probiotics Prevent Intestinal Barrier Dysfunction in Acute Pancreatitis in Rats via Induction of Ileal Mucosal Glutathione Biosynthesis (2009) PLoS ONE 4(2): e4512.

12. Siqin D, Wangv $\mathbf{C}$ et al. The key event of acute pancreatitis: Pancreatic duct obstruction and bile reflux, not a single one can be omitted. Med Hypotheses 2009; DOI:10.1016/j.hehy.2008.12.009.

13. Scheppach W. Abdominal compartment syndrome. Best Practice Res Clin Gastroenterol 2009; 23: 25-33.

14. Siebig S, Iesalnieks I, Bruennler T, Dierkes C, Langgartner J, Schoelmerich J, Wrede CE. Recovery from respiratory failure after decompression laparotomy for severe acute pancreatitis. World J Gastroenterol 2008; 14 (35): 5467-5470.

15. Zhang WF, Ni YL, Cai L, Li T, Fang XL, Zhang YT. Intraabdominal pressure monitoring in predicting outcome of patients with severe acute pancreatitis. Hepatobiliary Pancreat Dis Int 2007; 6: 420-423.

16. Dambrauskas Z, Parseliunas A, Gulbinas A, Pundzius J, Barauskas G. Early recognition of abdominal compartment syndrome in patients with acute pancreatitis. World J Gastroenterol 2009; 15 (6): 717-721.

17. Al-Bahrani AZ, Abid GH, Holt TA, McCloy RF, Benson J, Eddleston J, Ammori BJ. Clinical Relevance of Intra-Abdominal Hypertension in Patients with Severe Acute Pancreatitis. Pancreas 2008; $36: 39$.
18. De Waele JJ, Leppäniemi AK. Intra-Abdominal Hypertension in Acute Pancreatitis. World J Surg 2009; DOI 10.1007/s00268-009-9994-5.

19. Cicalese L, Sahai A, Sileri $P$ et al. Acute pancreatitis and bacterial translocation. Dig Dis Sci, 2001; 46: 1127-1132.

20. Rosas JM, Soto SN, Aracil JS et al. Intra-abdominal pressure as a marker of severity in acute pancreatitis. Surgery 2007; 141: 173-178.

21. De Waele JJ, De laet I, Malbrain ML. Rational intraabdominal pressure monitoring: how to do it? Acta Clin Belg 2007; 62: 16-25.

22. Malbrain ML, De Laet IE, De Waele JJ. IAH/ACS: The Rationale for Surveillance, World J Surg 2009; DOI 10.1007/s00268-009-0039-x.

23. Reis R, Labas P, Vician M, Ziak M. The abdominal compartment syndrome. Bratisl Lek Listy 2003; 104 (1): 32 - 35.

24. Sugure M, Bauman A, Jones F et al. Clinical examination is an inaccurate predictor of intraabdominal pressure. World J Surg 2002; 26 (12): 1428-1431.

25. Malbrain ML. Different techniques to measure intra-abdominal pressure (IAP): Time for a critical reapraisal. Intensive Care Med 2004; 30 (3): 357-371.

26. Malbrain ML, Chiumello D, Pelosi $P$ et al. Prevalence of intra-abdominal hypertension in critically ill patients: A multicentre epidemiological study. Intensive Care Med 2004; 30: 822-829.

27. Sanchez NC, Tenofsky PL, Dort JM et al. What is normal intraabdominal pressure? Am Surg 2001; 67 (3): 243-248.

28. Sugerman H, WindsorA, Bessos Metal. Intra-abdominal pressure, sagital abdominal diameterand obesity comorbidity. J Intern Med 1997;241 (1):71-79.

29. Caldwell CV, Ricotta JJ. Changes in visceral blood flow with elevated intraabdominal pressure. J Surg Res 1987; 43: 14-20.

30. Diebel LN, Dulchavsky SA, Brown WJ. Splanchnic ischaemia and bacterial translocation in the abdominal compartment syndrome. J Trauma 1997; 43: 852-855.

31. Ivatury RR, Cheatham ML, Malbrain ML, Sugrue M. Abdominal Compartment Syndrome, Landes Bioscience, USA, 2006, 308p.

32. Djavani K, Valtysson J, Björck M. Intra-abdominal hypertension (IAH) and outcome after abdominal aortic surgery. Abstract from the First World Congress on Abdominal Compartment Syndrome, Australia, 2004.

33. Keskinen P, Leppäniemi A, Pettila $V$ et al. Intra-abdominal pressure in acute necrotizing pancreatitis. Inaugural World Congress Abdominal Compartment Syndrome, Noosa, Australia 2004.

34. Lisi M, Sne B, Baillie F et al. Computed tomography guided percutaneous drainage of the abdominal cavity is a safe alternative to celiotomy in patients with abdominal compartment syndrome due to severe phlegmonous pancreatitis: a case report. Inaugural World Congress Abdominal Compartment Syndrome, Noosa, Australia, 2004.

35. Mifkovic A, Skultety J, Pindak D, Pechan J. Specific aspects of acute pancreatitis. Bratisl Lek Listy 2009; 110 (9): 544-552.

36. Jochberger S, Dörler J, Luckner G, Mayr VD, Wenzel V, Ulmer H, Morgenthaler NG, Hasibeder WR, Dünser MW. The vasopressin and copeptin response to infection, severe sepsis, and septic shock. Crit Care Med 2009; 37 (2): 476-482.

37. Morgenthaler NG. Copeptin: a biomarker of cardiovascular and renal function. Congest Heart Fail 2010; 16 Suppl 1: S37-44.

38. Katan M, Christ-Crain M. The stress hormone copeptin: a new prognostic biomarker in acute illness. Swiss Med Wkly 2010; 140: w13101.

Received January 18, 2011. Accepted December 17, 2012. 